\title{
Deformable Registration of Differently-Weighted Breast Magnetic Resonance Images
}

\author{
Tobias Boehler ${ }^{1}$, Sylvia Glasser ${ }^{2}$, Heinz-Otto Peitgen ${ }^{1}$ \\ ${ }^{1}$ Fraunhofer MEVIS, Bremen \\ ${ }^{2}$ Institut für Simulation und Graphik, Otto-von-Guericke-Universität Magdeburg \\ tobias.boehler@mevis.fraunhofer.de
}

\begin{abstract}
Dynamic breast magnetic resonance imaging (MRI) commonly acquires T1- and T2-weighted images. Joint inspection of both sequences in particular allows to distinguish between benign cyst-type and malignant lesions. However, in routine diagnostics both images are regarded independently, and immediate correlation is prevented by patient motion and tissue deformations. Differences in resolution and intensity distribution prevent a co-registration by established breast registration techniques. Instead, we propose to employ a deformable registration method that takes such differences into account. Accuracy of the method was confirmed through visual inspection and landmark-based assessment, indicating an average registration accuracy of $1.74 \mathrm{~mm}$. We demonstrate how the proposed technique facilitates a joint visualization of both MRI-sequences, thereby offering valuable diagnostic support.
\end{abstract}

\section{Introduction}

The role of breast magnetic resonance imaging is that of an adjunct modality for diagnostics. Although breast MRI has received an increased amount of attention over the last decades, it remains supplemental to conventional x-ray mammography (MG): sensitivity of breast MRI is high but its specificity is rather low, while being more cost-intensive than radiographic mammography [1]. MRI is therefore frequently consulted to confirm the malignity or benignity of lesions.

On the other hand, breast MRI allows to assess tissue-specific characteristics. While MG primarily images tissue density, breast MRI also enables functional and dynamic imaging. It is established practice to administer a ferro-magnetic contrast agent (typically Gadolinium-based) to accentuate tissue vascularity. The MR sequence of choice for this enhancement-visualization is the dynamic contrast-enhanced (DCE) T1-sequence which typically acquires up to ten images at distinct time points. Most workstation vendors allow to generate subtraction images, maximum-intensity projections thereof, as well as cine views showing all time points in succession. To remove spurious motion artifacts, a number of dedicated correction strategies for such images have been developed [2].

Contrarily, software support for T2-images is limited to a joint display with T1-series. Lesions are recovered by time-consuming manual spatial correlation of both images. A computerized exploitation of both intensity information is 
frequently prevented by motion artifacts. Existing motion correction strategies are often not readily applicable, as methods might assume identical characteristics for tissue intensities, tissue contrasts or image extents [2]. Particularly the difference in image resolutions might prevent such applications.

In this article we describe a deformable image registration method that aligns T1- and T2-images of the breast. To the best of our knowledge no registration method for this specific application has been proposed previously. The method respects differences in resolution, anisotropy and intensity characteristics. Accuracy of the method was estimated by a landmark-based quantitative estimation of target registration errors. As a result, the computed spatial deformation allows to superimpose T2-intensities on the anatomical image.

\section{Material and Methods}

Disagreement of T1- and T2-sequences of a single patient results primarily from patient motion. Unlike motion correction, sequence co-registration must account for the different image resolutions, as well as intensity distributions. The proposed registration scheme iteratively aligns a template image $T$ to a given, fixed reference image $R$. Regularity of the solution is enforced by the linear-elastic operator [3]. A finite-impulse response approximation of this operator is employed, exploiting its eigenfunction representation [4]. Derivation of the filter response yields a non-symmetric, discrete filter kernel, and iterative convolution of a displacement field $U$ with this kernel explicitly enforces linear-elastic regularity. For the proposed registration, a $7 \times 7 \times 7$ filter kernel is employed, with $\mu=500$ and $\lambda=0.1$ set as elasticity moduli.

The similarity of $R$ and deformed $\hat{T}$ is estimated by the local cross correlation (LCC) distance measure $D(R, \hat{T}) \in \mathbb{R}$, which exhibits robustness w.r.t. intensity variations [5]. The LCC measure is calculated in a 6-neighborhood around each reference voxel, and locations for $\hat{T}$ are mapped in device coordinate space independent of actual image resolution and voxel sizes. Updates of the deformation field are estimated as first order derivatives of the distance function, resembling artificial body forces. This update is regularized with the elastic kernel and composed with previous deformations, similar to the demon's algorithm scheme [6]. Registration is an iterative two-step process of force estimation and regularization, where the updated deformation at iteration $k$ is composed as

$$
U^{k+1}=K *\left[U^{k} \circ \rho \gamma U_{\text {update }}^{k}\right]
$$

and where $K$ is the aforementioned regularization kernel. The $\circ$ operator denotes the displacement field warping that composes the two corresponding image transformations. Line search is performed in each iteration, starting with a predefined step width $\rho \in \mathbb{R}$ that is consecutively being reduced by a factor $\gamma \in \mathbb{R}$ until a reduced error value is determined or the maximum number of search steps has been reached. The resulting deformation field is then applied to the original template image using linear intensity interpolation. Convergence is declared when the distance measure satisfies a set of termination criteria. A multi-resolution 
scheme is used to increase capture range and reduce computational complexity. For both regularization and force estimation, Neumann-0 boundary conditions are set in order to enable flexible boundaries. The implementation furthermore exploits multi-threading to increase computational performance.

\section{Results}

Initial validation of the proposed registration method was carried out on 10 clinical dynamic T1-weighted and T2-weighted MR images acquired with a Philips Panorama MRI device. Both in-plane and out-of-plane resolution had been adjusted for each dataset, varying from $432^{2} \times 95$ voxels to $560^{2} \times 107$ voxels, and $0.65^{2} \times 1.5 \mathrm{~mm}$ to $0.67^{2} \times 1.5 \mathrm{~mm}$ for the T1-images, as well as $528^{2} \times 53$ to $640^{2} \times 57$ voxels and $0.53^{2} \times 3.0 \mathrm{~mm}$ to $0.59^{2} \times 3.0 \mathrm{~mm}$ for the T2-images. Generally, both in-plane resolution and slice-thickness are lower for T1-weighted images. As discussed previously, it is therefore mandatory to incorporate actual voxel extent to ensure accurate registration. The dynamic T1-series contained five time points with $75 \mathrm{~s}$ intervals between individual acquisitions. For the T1sequence, a gradient-echo sequence with $\mathrm{TR}=11.42 \mathrm{~ms}, \mathrm{TE}=6.0 \mathrm{~ms}$ and flip angle $25^{\circ}$ was employed. No fat-suppression had been selected. T2-weighted imaging used a spin-echo sequence with $\mathrm{TR}=7500 \mathrm{~ms}, \mathrm{TE}=140 \mathrm{~ms}$ and flip angle of $90^{\circ}$.

\subsection{Visual assessment}

Inspection of original and deformed images allows to investigate image consistency. In order to compare different intensities of the T1- and T2-images, isocontours are overlaid (Fig. 1). Contours are generated from T1-intensities and superimposed on original and registered T2-images, focused in selected fieldsof-view to highlight local differences. The actual visual inspection performed on-screen displayed the entire image information, which is vital to assess registration quality in remote regions, e.g., axillary lymph node locations.

In addition to contour projections, the corresponding portions of the displacement field are displayed as corresponding vectors in the rightmost column, with each seventh vector shown. By magnitude, the displayed images contained the largest displacements and therefore most pronounced image perturbations. It is vital to ensure that larger deformations retain deformation regularity and consistency, which is only assessable through the generated deformation field.

\subsection{Landmark distances}

Quantitative validation of the described method was achieved through measurement of target registration errors [7]. For each pair of T1- and T2-images at least three corresponding pseudo-landmarks at characteristic positions were placed by an expert breast radiologist. Euclidean distances between point pairs were estimated (Tab. 1). 
Table 1. Mean landmark distances estimated for all evaluation datasets. Left to right: mean distance before $\left(d_{\text {pre }}\right)$ and after registration $\left(d_{\text {post }}\right)$, remaining absolute $\left(i_{\text {abs }}\right)$ and relative distances $\left(i_{\text {rel }}\right)$. Both standard deviations and distances are reduced.

\begin{tabular}{lllll}
\hline Distance & $d_{\text {pre }}[\mathrm{mm}]$ & $d_{\text {post }}[\mathrm{mm}]$ & $i_{\text {abs }}[\mathrm{mm}]$ & $i_{\text {rel }}(\%)$ \\
\hline Mean & 2.86 & 1.74 & 1.12 & 71.11 \\
Std.dev. & 1.38 & 1.00 & 1.11 & 45.93 \\
\hline
\end{tabular}

\section{Discussion}

Using the proposed registration method a precise, accurate alignment of T1and T2-images is accomplished. Visual inspection of transformed images and corresponding displacement fields confirms an increased tissue alignment. The results indicate that actual breast deformations are inherently non-linear, as the local magnitude of displacements varies over the entire imaged breast (Fig. 1). Deformation fields were smooth and folding-free.

Quantitative evaluation of landmark errors shows that the mean distance is reduced from $2.86 \pm 1.38 \mathrm{~mm}$ to $1.74 \pm 1.00 \mathrm{~mm}$. Relative remaining distances are reduced to $71.11 \%$ of their original value, while associated standard deviation reaches $45.93 \%$. It should be noted that the distance assessment considered misplaced landmark positions. Disregard of such inaccurate placements would result in even smaller remaining distances, albeit causing a validation bias. An
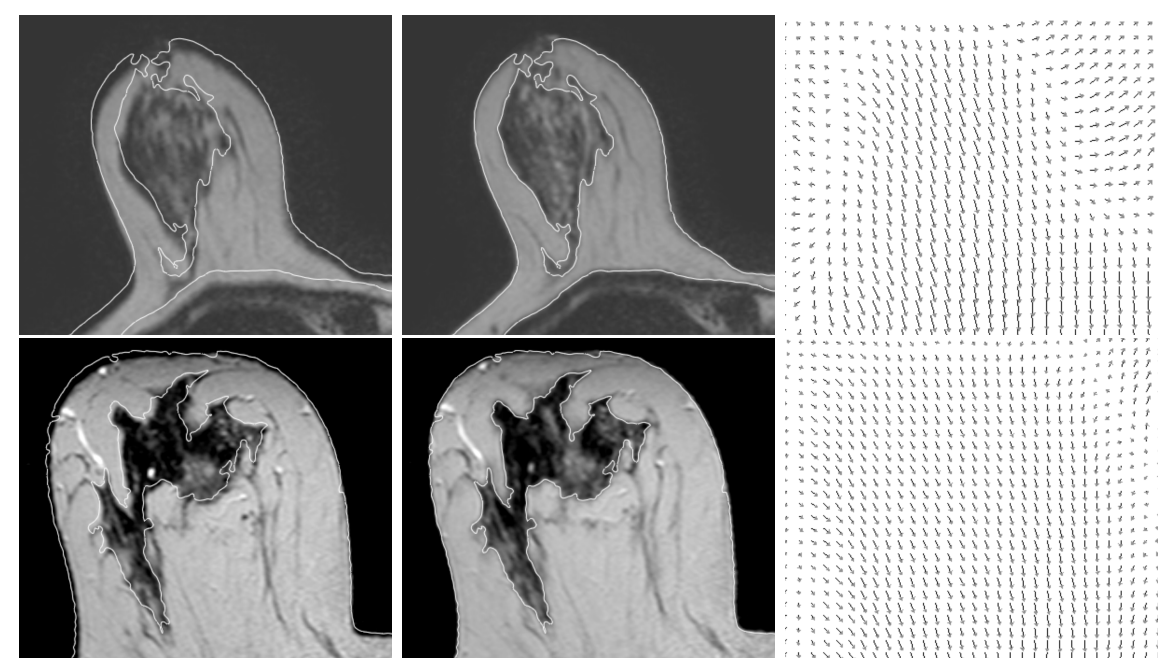

Fig. 1. Example magnified registration results: T1-references are overlaid as white iso-contours, both for the original (left) and registered (middle) configurations. Note the improved agreement of tissue boundaries with the iso-contour at T1-tissue borders. Right column: corresponding displacements. Images courtesy Uta Preim, Magdeburg. 
Fig. 2. Joint visualization of $\mathrm{T} 1$ - and $\mathrm{T} 2$-images, left to right: original images (slices $42 / 19$ ), subtraction of two dynamic time points, as well as overlay of the registered T2-image on the dynamic subtraction image. Note the characteristic hyper-intense $\mathrm{T} 1$-appearance of the lesion and its hypo-intense T2-intensities, indicating malignity.

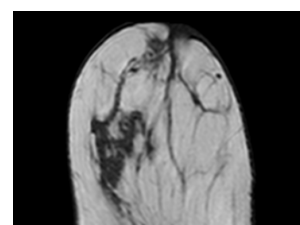

(a) T1-image

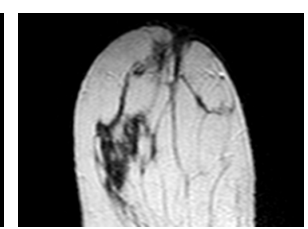

(b) T2-image

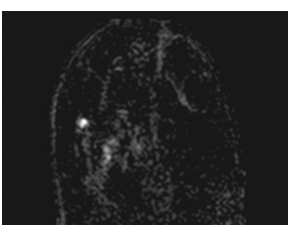

(c) T1 subtraction

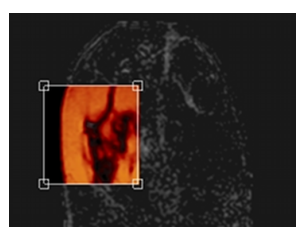

(d) T2-overlay

initial joint visualization is shown in Fig. 2: after registration of T1- and T2weighted MR-images, the latter is overlaid on the difference image computed from two time points of the dynamic MRI. A region of interest is chosen interactively, offering the radiologist an immediate and intuitive access to T2-data. Using T1- and T2-data, the enhancing lesion is identified as being malignant.

In summary, the proposed method allows to efficiently align T1- and T2images with sub-voxel precision, thereby facilitating a joint analysis of both sequences. A preliminary joint visualization was presented. Currently, further quantitative evaluation and an assessment of the joint visualization are conducted.

Acknowledgement. The authors would like to thank Dr. Uta Preim, University Hospital Magdeburg, for providing both images and annotations. Parts of this work have been funded as part of the EU-project HAMAM, FP7-ICT, grant no. 224538. Sylvia Glasser is part of the project "Efficient Visual Analysis of Dynamic Medical Image Data" supported by the DFG Priority Program 1335: "Scalable Visual Analytics" .

\section{References}

1. Orel SG, Schnall MD. MR imaging of the breast for the detection, diagnosis, and staging of breast cancer. Radiology. 2001;220(1):13-30.

2. Guo Y, et al. Breast image registration techniques: a survey. Med Biol Eng Comput. $2006 ; 44: 15-26$.

3. Modersitzki J. Numerical Methods for Image Registration. New York: Oxford University Press; 2004.

4. Gramkow C, Bro-Nielsen M. Comparison of three filters in the solution of the Navier-Stokes equation in registration. In: Proc SCIA; 1997. p. 795-802.

5. Netsch T, et al. Towards real-time multi-modality 3-D medical image registration. In: Proc IEEE ICCV; 2001. p. 718-25.

6. Vercauteren $\mathrm{T}$, et al. Non-parametric diffeomorphic image registration with the demons algorithm. Lect Notes Computer Sci. 2007;4792:319-26.

7. Hajnal JV, Hawkes DJ, Hill DLG. Medical Image Registration. CRC Press; 2001. 